ISSN: 2224-0616

Int. J . Agril. Res. Innov. \& Tech. 4 (1): 77-87, J une, 2014

Available online at http:// www.ijarit.webs.com

\title{
GENETIC DIVERSITY ANALYSIS OF RICE (Oryza sativa L.) LANDRACES THROUGH RAPD MARKERS
}

\author{
M.S. Alam ${ }^{*}$, S.N. Begum², R. Gupta ${ }^{3}$ and S.N. Islam
}

Received 7 March 2014, Revised 6 June 2014, Accepted 28 June 2014, Published online 30 June 2014

\begin{abstract}
The molecular marker is a useful tool for assessing genetic variations and resolving cultivar identities. Information on genetic diversity and relationships among rice landraces from Bangladesh is currently very limited. Thirty-five rice genotypes including 33 landraces and $01 \mathrm{HYV}$ of Bangladesh and 1 Indian landrace of particular interest to breeding programs were evaluated by means of random amplified polymorphic DNA (RAPD) technique. For molecular characterization, RAPD markers viz, OPC 03, OPC 04 and OPA 01 gave reproducible and distinct polymorphic amplified products. A total of 20 RAPD bands were scored of which 15 polymorphic amplification products were obtained by using these arbitrary primers. The size of amplified fragments were ranged from 550 to $1775 \mathrm{bp}$. Based on analysis performed on a similarity matrix using UPGMA, 35 genotypes were grouped into 2 main clusters. Landrace Sylhet balam and Mota aman was totally different from other genotypes. The information will facilitate selection of genotypes to serve as parents for effective rice breeding programs in Bangladesh.
\end{abstract}

Keywords: Crop Diversity, Characterization, Genetic Distance, Genetic Identity, Polymorphic Loci

${ }^{1}$ Department of Genetics and Plant Breeding, Bangladesh Agricultural University, Mymensingh, Bangladesh.

${ }^{2}$ Senior Scientific Officer, Plant Breeding Division, Bangladesh Institute of Nuclear Agriculture, Mymensingh, Bangladesh.

${ }^{3}$ Scientific Officer, Bangladesh Institute of Nuclear Agriculture, Sub Station, Khagrachari, Bangladesh.

*Corresponding author's email: agt.shahed@gmail.com, m.s.alam@irri.org (M.S. Alam)

\section{Introduction}

Rice (Oryza sativa L.) diversity consists of landraces, improved cultivars, hybrids and closely related wild relatives. Landraces are local crop varieties developed in primitive agricultural system, rather than being deliberately bred, selected by the farmers over many generations. Landraces of rice played a very important role in the local food security and sustainable development of agriculture, in addition to their significance as genetic resource for rice genetic improvement. To maintain crop diversity, collection, characterization and conservation of traditional landraces are vital. Bangladesh is a good source of landraces of rice. To formulate a sustainable breeding program precise knowledge about genetic divergence for yield components is a crucial one as varietal improvement depends mainly on the selection of parents with high genetic divergence in hybridization that is supposed to increase the chance of obtaining maximum heterosis and give broad spectrum of variability in segregating generations. PCR-based molecular marker technique RAPD analysis is a reliable tool for assessing genetic diversity. This technique use a single short oligonucleotide primer (9-10 bp) of arbitrary DNA sequence and polymerase chain reaction (PCR) mediated amplification of random fragment from genomic DNA. So, among the available DNA molecular techniques, RAPD has many advantages over others such as ease and rapidity of analysis, a relatively low cost, availability of large numbers of primers and the requirement of a very small amount of DNA for analysis (Weising et al., 1995). Advantages associated with RAPDs have made them a favorite marker technique in mapping, the determination of phylogenetic relationships, genetic diversity, and identification of cultivars and parents in a number of plant species. Since the RAPD technique involves enzymatic amplification of target DNA by PCR using arbitrary primers, it is also called Arbitrarily Primed Polymerase Chain Reaction (AP-PCR) or DNA Amplification Fingerprinting (DAF). RAPD markers tend to estimate intra or inter genetic distances more distantly related individuals. In addition, no prior knowledge of sequence is required. Since primers can be chosen arbitrarily, any organism can be mapped with the same set of primers. These advantages make RAPD markers far easier to work. The objective of this present study was to evaluate genetic divergence of 35 rice genotypes with three RAPD markers. 


\section{Materials and Methods}

The experiment was carried out at the Biotechnology Laboratory, Biotechnology Division, Bangladesh Institute of Nuclear Agriculture, Mymensingh, during July 2012 to December 2012.

\section{Plant materials}

Thirty five rice genotypes including 33 landraces (i.e. Hati bajore, Malagoti, Kuchra, Enghi, J amai naru, Hari, Dakh shail, Moina moti, Marish shail, Patnai, Bhute shallot, Kute patnai, Moghai balam, Sada gotal, Khak shail, Jota balam, Khainol, Hamai, Sylhet balam, Mota aman, Ghigoj, Piarjat, Lal biroi, Lalanamia, Golapi, Asam binni, Kakua binni, Ledra binni, Rotisail, Genggeng binni, Jolkumri, Mowbinni and Bogi) and 1 HYV of Bangladesh (i.e. Binadhan-8) and 1 Indian landrace (i.e. Nona bokhra) were used for genetic diversity analysis through RAPD markers.

\section{DNA extraction}

In order to carry out RAPD analysis, young, vigorously growing fresh leaf samples were collected from 25 days old seedling of each genotype and used as the source of genomic DNA. DNA was extracted from the leaves of each genotype using the Cetyl Trimethyl Ammonium Bromide (CTAB) mini-prep method. The simplified mini scale procedure for DNA isolation in PCR analysis developed at IRRI was followed. Confirmation of the DNA was done through electrophoresis on a $0.8 \%$ agarose gel and DNA quantification was through the spectrophotometer's (spectronic ${ }^{@}$ Genesisr ${ }^{\mathrm{TM}}$ ). Ten primers of random sequence were screened for amplification of the DNA sequences. Primers resulting in faint or irreproducible bands were excluded from subsequent analysis. A final subset of seven primers exhibiting good quality banding patterns and sufficient variability from where finally three primers were selected for further analysis.

\section{PCR reaction and electrophoresis}

PCR reactions were performed on each DNA sample in a 10 $\mathrm{\mu l}$ reaction mix containing $1 \mu \mathrm{l}$ Ampli Taq polymerase buffer (10X), $2.5 \mathrm{\mu l}$ Primer $(10 \mu \mathrm{M}), 1 \mu \mathrm{l} d N T P s(250 \mu \mathrm{M}), 0.2 \mu \mathrm{l}$ Ampli Taq DNA polymerase and $3.3 \mu \mathrm{l}$ sterile deionized water. Two $\mathrm{\mu l}$ genomic DNA was added and finally, total volume was made $10 \mu \mathrm{l}$. DNA amplification was performed in an oil-free thermal cycler. The reaction mix was preheated at $94^{\circ} \mathrm{C}$ for 3 minutes followed by 40 cycles of 1 min denaturation at $94^{\circ} \mathrm{C}, 1 \mathrm{~min}$ annealing at $54^{\circ} \mathrm{C}$ and elongation or extension at $72^{\circ} \mathrm{C}$ for 2 minutes. After the last cycle, a final step for 7 minutes at $72^{\circ} \mathrm{C}$ to allow complete extension of all amplified fragments. After completion of cycling program, reactions were held at $4^{\circ} \mathrm{C}$. Electrophoresis was carried out in 0.5 X TBE buffer on a $1.5 \%$ agarose gel and amplified fragments were visualized by staining with ethidium bromide.

\section{Data analysis}

The amplified bands were visually scored as present (1) and absent (0) separately for each individual primer. The scores of bands were pooled to create a single data matrix. This was used to estimate polymorphic loci, Nei's (1972) gene diversity, population differentiation (Gst), gene flow $\left(\mathrm{N}_{\mathrm{m}}\right)$. Genetic distance (GD) and to construct a UPGMA (Unweighted Pair Group Method of Arithmetic Means) dendrogram among populations using a computer program, POPGENE (Version 1.31); (Yeh et al., 1997).

\section{Results and Discussion}

\section{RAPD banding pattern}

Ten primers were initially screened for their ability to produce polymorphic patterns and out of 10, three primers viz., OPC 03, OPC 04 and OPA 01 gave reproducible and distinct polymorphic amplified products. DNA amplification from all the primers tested in this study was not consistently reproducible, is a very common feature of RAPD technique. A total of 20 RAPD bands were scored of which 15 (73.61\%) polymorphic amplification products were obtained by using these arbitrary primers. This proportion of polymorphism was similar compared to previous RAPD analysis in rice genotypes by Skaria et al. (2011) who obtained $72.27 \%$ of polymorphic products. The size of the amplification products ranged from 550-1775 bp (Table 1). The selected 3 primers produced comparatively maximum number of high intensity band with minimal smearing, good technical resolution and sufficient variation among different cultivars. The dissimilar numbers of bands were generated by primer OPC 03, OPC 04 and OPA 01. Besides, the primer OPC-03 amplified maximum number of polymorphic bands (100\%) while the primer OPA-01 generated the least (33.33\%) polymorphic bands which were minimal in number. The banding patterns of 35 rice using primers OPC 03, OPC 04 and OPA 01 are shown in Figs. 1, 2 and 3, respectively. The DNA polymorphisms were detected according to the presence and absence of band. Absence of band may be caused by failure of primer to anneal a site in some individuals due to nucleotide, sequences difference or by insertions or deletions between primer sites (Clark and Lanigan, 1993). Frequencies of polymorphic loci (RAPD markers) in 35 rice genotypes were presented in Table 2. 
Table 1. RAPD primers with corresponding bands score and their size range together with polymorphic bands observed in 35 rice genotypes

\begin{tabular}{lccccc}
\hline $\begin{array}{l}\text { Primer } \\
\text { code }\end{array}$ & $\begin{array}{c}\text { Sequences } \\
\left(5^{\prime}-3^{\prime}\right)\end{array}$ & $\begin{array}{c}\text { Total } \\
\text { number of } \\
\text { bands } \\
\text { scored }\end{array}$ & $\begin{array}{c}\text { Size ranges } \\
(\mathrm{bp})\end{array}$ & $\begin{array}{c}\text { Number of } \\
\text { polymorphic bands }\end{array}$ & $\begin{array}{c}\text { Proportion of } \\
\text { polymorphic } \\
\text { loci (\%) }\end{array}$ \\
\hline OPC 03 & GGGGGTCTT & 6 & $550-1650$ & 6 & 100.00 \\
OPC 04 & CCGCATCTAC & 8 & $600-1700$ & 7 & 87.50 \\
OPA 01 & CAGGCCCTTC & 6 & $600-1775$ & 2 & 33.33 \\
\hline Total & & 20 & & 15 & 220.83 \\
\hline Average & & 6.67 & & 5.00 & 73.61 \\
\hline
\end{tabular}

\section{Genetic variation}

The values of Nei's (1973) gene diversity and Shannon's information index for different accessions of rice across all loci are shown in Table 2. The estimate of Nei's (1973) genetic diversity for 35 genotypes of rice was 0.12 and
Shannon's information index was 0.22. There was a high level of genetic variation among the studied genotypes of rice from the proportion of polymorphic loci point of view.

Table 2. Summary of Frequencies of polymorphic gene, genetic diversity statistics and Shanon information index for all loci in 35 rice genotypes

\begin{tabular}{cccc}
\hline Loci & Gene frequency & Gene diversity $(\mathrm{h})$ & Shanon information index (i) \\
\hline OPC 03-1 & 0.8000 & 0.3200 & 0.5004 \\
OPC 03-2 & 0.8857 & 0.2024 & 0.3554 \\
OPC 03-3 & 0.9429 & 0.1078 & 0.2190 \\
OPC 03-4 & 0.9429 & 0.1078 & 0.2190 \\
OPC 03-5 & 0.9714 & 0.0555 & 0.1297 \\
OPC 03-6 & 0.9143 & 0.1567 & 0.2925 \\
OPC 04-1 & 0.7714 & 0.3527 & 0.5375 \\
OPC 04 - & 0.6000 & 0.4800 & 0.6730 \\
OPC 04 -3 & 0.9714 & 0.0555 & 0.1297 \\
OPC 04 -4 & 0.9714 & 0.0555 & 0.1297 \\
OPC 04 -5 & 0.0000 & 0.0000 & 0.0000 \\
OPC 04 -6 & 0.2286 & 0.3527 & 0.5375 \\
OPC 04 - & 0.9714 & 0.0555 & 0.1297 \\
OPC 04 -8 & 0.9714 & 0.0555 & 0.1297 \\
OPA 01-1 & 0.0000 & 0.0000 & 0.0000 \\
OPA 01-2 & 0.0857 & 0.1567 & 0.2925 \\
OPA 01-3 & 0.0000 & 0.0000 & 0.0000 \\
OPA 01-4 & 0.0000 & 0.0000 & 0.0000 \\
OPA 01-5 & 0.0000 & 0.0000 & 0.0000 \\
OPA 01-6 & 0.0286 & 0.0555 & 0.1297 \\
\hline Mean & & 0.1285 & 0.2203 \\
\hline St. Dev. & & 0.1420 & 0.2059 \\
\hline
\end{tabular}

\section{Genetic distance and genetic identity}

Pair-wise comparisons of Nei's (1972) genetic distance (GD) between rice genotypes were computed from combined data for the three primers and the values ranged from 0.0000 to 0.9000 ( Table 3). Comparatively higher genetic distance was observed between the genotypes Golapi vs. Bogi. 


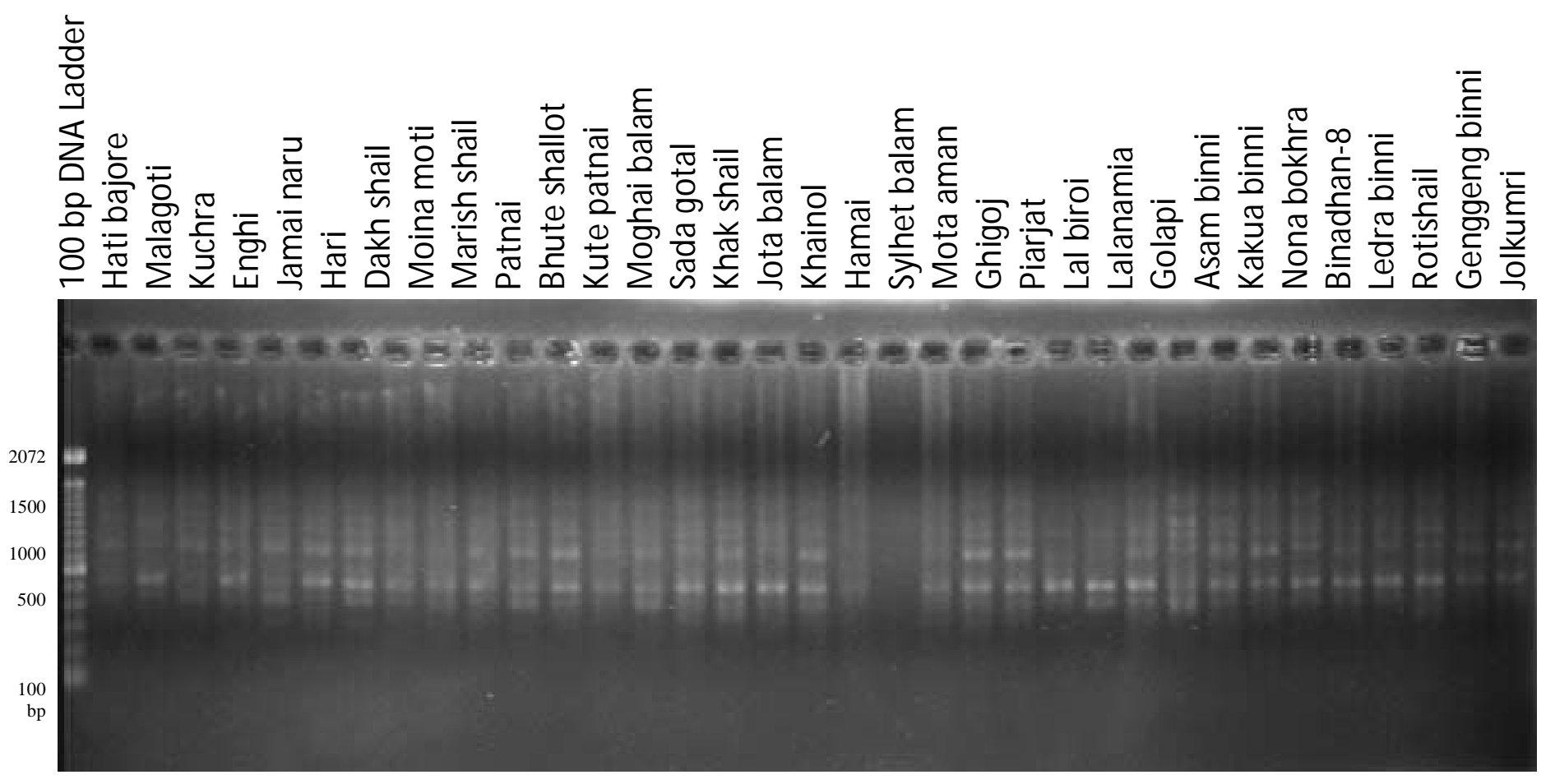

Fig. 1. RAPD profiles of different 35 rice genotypes using primer OPC-03 


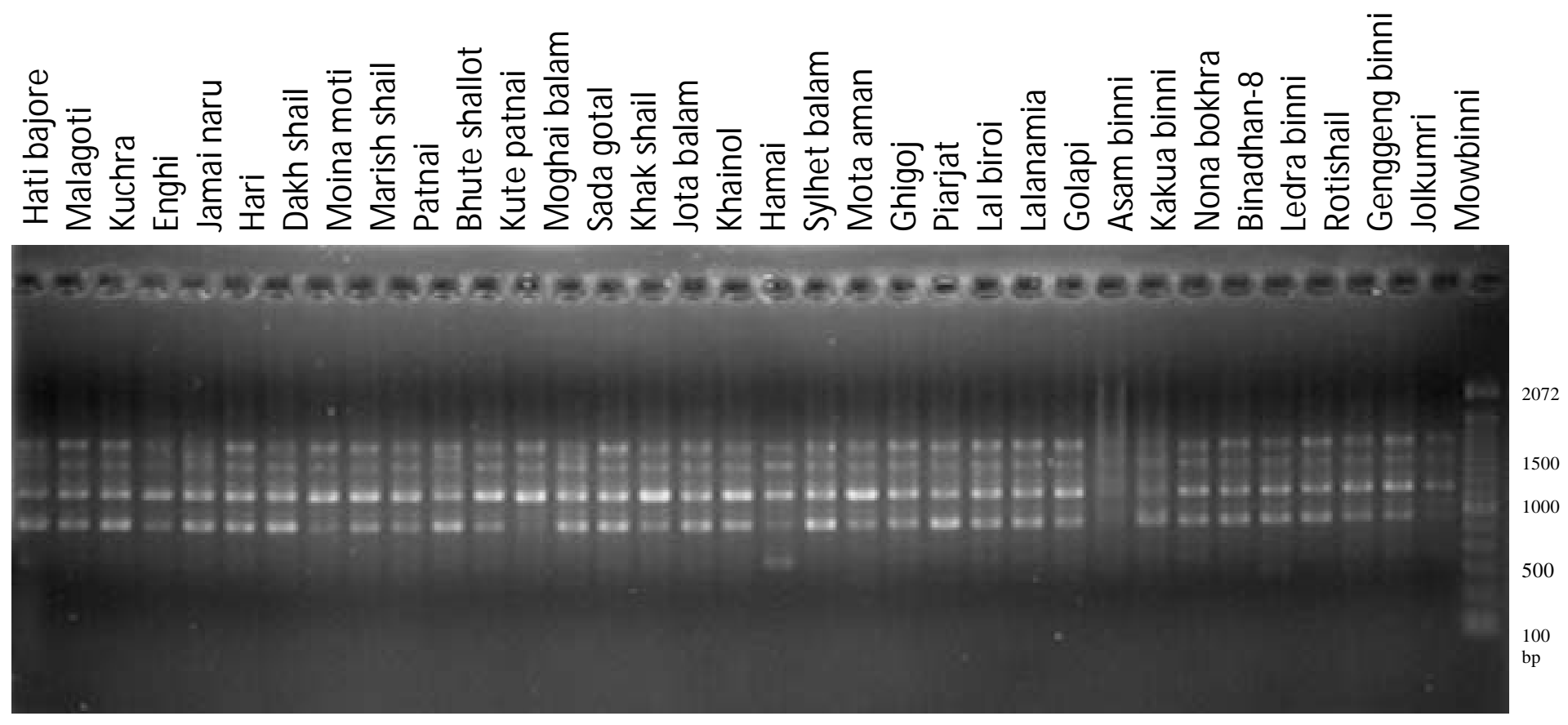

Fig. 2. RAPD profiles of different 35 rice genotypes using primer OPC-04 


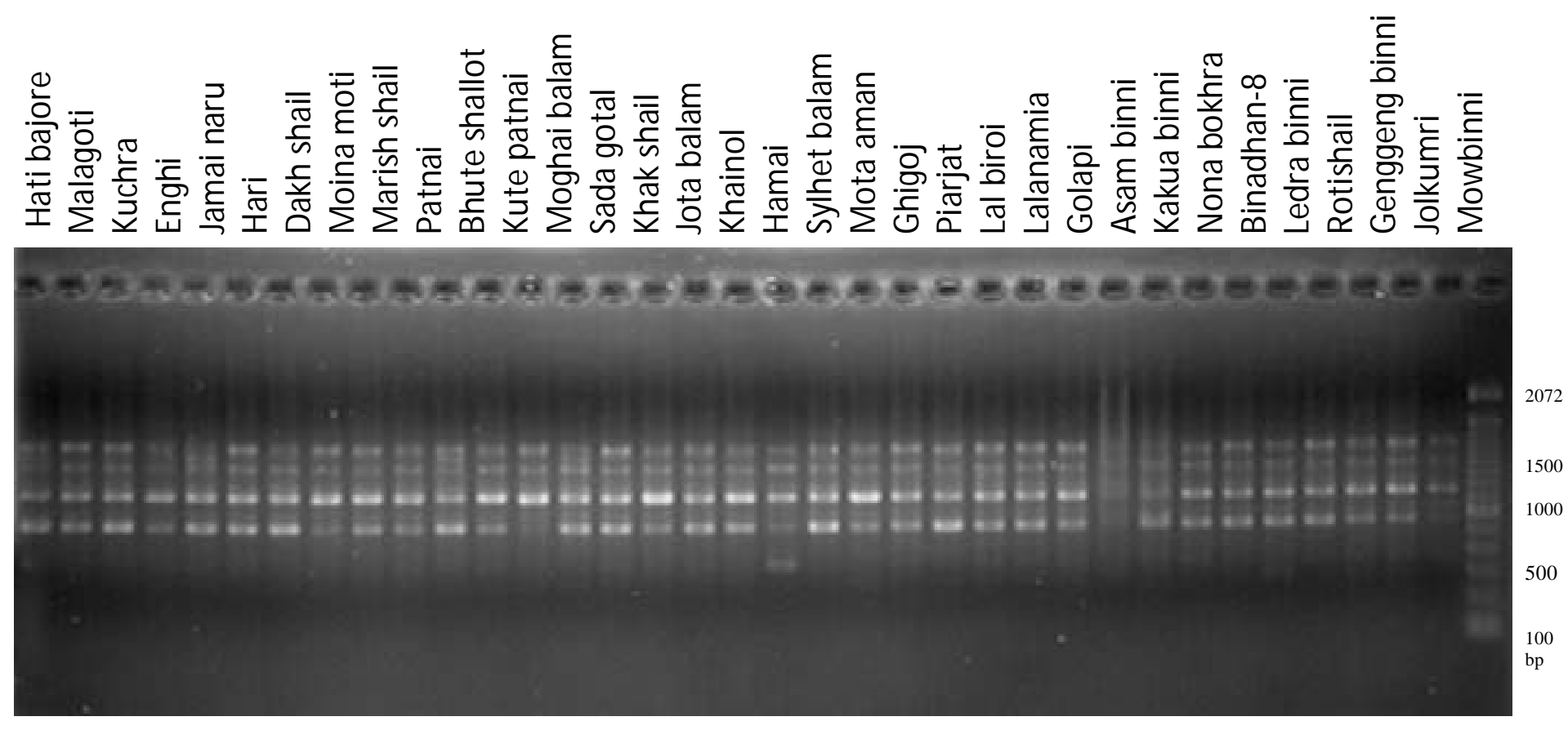

Fig. 3. RAPD profiles of different 35 rice genotypes using primer OPA-01 
Table 3. Summary of Nei's genetic identity (above diagonal) and genetic distance (below diagonal) between 35 rice genotypes

\begin{tabular}{|c|c|c|c|c|c|c|c|c|c|c|c|c|c|c|c|c|c|c|}
\hline Acce. & 1 & 2 & 3 & 4 & 5 & 6 & 7 & 8 & 9 & 10 & 11 & 12 & 13 & 14 & 15 & 16 & 17 & 18 \\
\hline 1 & **** & 0.9500 & 1.0000 & 0.9500 & 0.9000 & 0.9500 & 0.9000 & $\overline{0.9000}$ & 0.9000 & 0.9500 & 0.8500 & 0.9000 & 0.9500 & 0.8500 & 0.9000 & 0.9000 & 0.9000 & 0.9500 \\
\hline 2 & 0.0513 & $* * * *$ & 0.9500 & 0.9000 & 0.8500 & 1.0000 & 0.8500 & .8500 & & 0.9000 & 0.8000 & 0.8500 & 0.9000 & 0.8000 & 0.8500 & 0.8500 & & \\
\hline 3 & 0000 & .0513 & $* * * *$ & 0.9500 & 0.9000 & 0.9500 & 0.9000 & .9000 & 0.9000 & 0.9500 & 0.8500 & 0.9000 & 0.9500 & 0.8500 & 0.9000 & 0.9000 & 0.9000 & 0.9500 \\
\hline 4 & .0513 & 0.1054 & 0.0513 & $* * * *$ & 0.9500 & 0.9000 & 0.9500 & .9500 & 0.8500 & 0.9000 & 0.9000 & 0.9500 & 0.9000 & .9000 & 0.9500 & 0.9500 & & 0.9000 \\
\hline 5 & 1054 & 1625 & 1054 & 0.0513 & $* * * *$ & 0.8500 & 0.9000 & 9000 & 0.8000 & 0.8500 & 0.9500 & 0.9000 & 8500 & & 0.9000 & 0.9000 & 0.8000 & 0.8500 \\
\hline 6 & .0513 & 0000 & 0.0513 & 0.1054 & 0.1625 & $* * * *$ & 0.8500 & 0.8500 & 0.9500 & 0.9000 & 0.8000 & 0.8500 & 0.9000 & 0.8000 & 0.8500 & 0.8500 & 0.9500 & 0.9000 \\
\hline 7 & 1054 & & 1054 & 0513 & 0.1054 & 0.1625 & & 1.0000 & 0.9000 & 0.9500 & & & & & & & & \\
\hline 8 & 0.1054 & 0.1625 & 0.1054 & 0.0513 & 0.1054 & 0.1625 & 0.0000 & $* * * *$ & 0.9000 & 0.9500 & 0.9500 & 1.0000 & 0.9500 & 0.9500 & 1.0000 & 1.0000 & 0.9000 & 0.9500 \\
\hline 9 & & & 0.1054 & & 0.2231 & & 0.1054 & 0.1054 & $* * * *$ & 0.9500 & 0.8500 & & & & & & & \\
\hline 10 & 0513 & 1054 & 0.0513 & 0.1054 & 0.1625 & 0.1054 & 0.0513 & 0.0513 & 0.0513 & $* * * *$ & 0.9000 & 0.9500 & 1.0000 & 0.9000 & 0.9500 & 0.9500 & 500 & 1.0000 \\
\hline 11 & 1625 & 2231 & 0.1625 & 0.1054 & 0.0513 & 0.2231 & & & & 0.1054 & $* * * *$ & 0.9500 & 0.9000 & 1.0000 & 0.9500 & 0.9500 & & 0.9000 \\
\hline 12 & 1054 & & 1054 & 0.0513 & & & 0.0 & & & & 0.0513 & **** & 0.9500 & & & & & 0.9500 \\
\hline 13 & 0513 & 0.1054 & 0.0513 & 0.1054 & 0.16 & 0.1054 & 0.0 & & 0.0513 & & & 0.0513 & & 0.9000 & 0.9500 & 0.9500 & 0.9500 & 1.0000 \\
\hline 14 & 1625 & 2231 & 0.1625 & 0.1054 & 0.05 & 0.2231 & & & & 0.1054 & & & 0.1054 & $* * * *$ & 0.9500 & 0.9500 & 0.8500 & 0.9000 \\
\hline 15 & 1054 & 0.1625 & 0.1054 & 0.0513 & 0.10 & 0.16 & 0.0 & & & 0.0 & & & & 0.0513 & $* * * *$ & 1.0000 & 000 & 0.9500 \\
\hline 16 & 1054 & 0.1625 & 0.1054 & 0.0513 & 0.1054 & 0.1625 & 0.00 & 00 & 0.1054 & 0.0 & 0.0 & 0.0 & & 0.0513 & 0.0000 & & 0.9000 & 0.9500 \\
\hline 17 & 1054 & 0513 & 0.1054 & 0.16 & & & 0.1 & & 0.0000 & & & & & & & 0.1054 & $* * * *$ & 0.9500 \\
\hline 18 & & & & & & & & & & & & & & & & & & $* * * *$ \\
\hline 19 & 4308 & 5108 & 4308 & 67 & 08 & & & & & 0.5 & 0.5 & 0.4 & 0.5108 & & 0.4 & 308 & 978 & 0.5108 \\
\hline 20 & 0.4308 & & 0.4308 & 0.5 & & & & & & & & & & & & & & 0.3567 \\
\hline 21 & 0513 & 1054 & 0.0513 & 0.1054 & 0.16 & 0.10 & & & & 00 & & & 000 & 0.1054 & & 513 & & 0.0000 \\
\hline 22 & & & & & & & & & & & & & & & & & & 000 \\
\hline 23 & & 1054 & 16 & & & 0.10 & & & & & & & & & & & & 0.1054 \\
\hline 24 & & & 25 & 0 & & & & & & & & & & & & & & 0.1054 \\
\hline 25 & & & 0.10 & 0.16 & & & & & & & & & & & & & & \\
\hline & & 0 & & & & & & & & & & & & & & & & 0.0513 \\
\hline 27 & & & & & & & & & & & & & & & & & & .0513 \\
\hline 28 & & & 0.1054 & & & & & & & & & & & & & & & \\
\hline & & 0.10 & 0.05 & & & & & & & & & & & & & & & \\
\hline 30 & 0.1054 & 0.1625 & 0.1054 & 0.0513 & 0.1054 & 0.1625 & 0.0000 & 0.0000 & 0.1054 & 0.0513 & 0.0513 & 0.0000 & 0.0513 & 0.0513 & 0.0000 & 0.0000 & 0.1054 & 0.0513 \\
\hline & & & 0.2877 & & & & & & & & & & & & & & & 0.3567 \\
\hline 32 & & & 0.0513 & & & & & & & & & & & & & 513 & 0.0513 & .0000 \\
\hline & 0.0513 & 0.1054 & 0.0513 & 0.1054 & 0.1625 & 0.1054 & 0.0 & 0.0513 & & 0.0000 & 0.10 & 0.0 & 0.0000 & 0.1054 & & & 0.0513 & 0000 \\
\hline & & 0.1625 & 0.2231 & 0.2877 & 0.3 & 0.1625 & & & 0.1054 & 0.1625 & 0.2877 & 0.2231 & 0.1625 & 0.2877 & 0.2 & 231 & 054 & 0.1625 \\
\hline & & & & & & .1625 & & & 0.1054 & & $.28 / 1$ & .2231 & 0.1625 & $0.28 / 1 /$ & 0.2231 & 0.2231 & 0.1054 & 0.1025 \\
\hline
\end{tabular}


Table 3. Contd.

\begin{tabular}{|c|c|c|c|c|c|c|c|c|c|c|c|c|c|c|c|c|c|}
\hline Acce. & 19 & 20 & 21 & 22 & 23 & 24 & 25 & 26 & 27 & 28 & 29 & 30 & 31 & 32 & 33 & 34 & 35 \\
\hline 1 & 0.6500 & 0.6500 & 0.9500 & 0.9500 & 0.8500 & 0.8500 & 0.9000 & 0.9000 & 0.9000 & 0.9000 & 0.9500 & 0.9000 & 0.7500 & 0.9500 & 0.9500 & 0.8000 & 0.8000 \\
\hline 2 & 0.6000 & 0.6000 & 0.9000 & 0.9000 & 0.9000 & 0.8000 & 0.8500 & 0.9500 & 0.8500 & 0.8500 & 0.9000 & 0.8500 & 0.7000 & 0.9000 & 0.9000 & 0.8500 & 0.8500 \\
\hline 3 & 0.6500 & 0.6500 & 0.9500 & 0.9500 & 0.8500 & 0.8500 & 0.9000 & 0.9000 & 0.9000 & 0.9000 & 0.9500 & 0.9000 & 0.7500 & 0.9500 & 0.9500 & 0.8000 & 0.8000 \\
\hline 4 & 0.7000 & 0.6000 & 0.9000 & 0.9000 & 0.8000 & 0.8000 & 0.8500 & 0.8500 & 0.9500 & 0.9500 & 0.9000 & 0.9500 & 0.8000 & 0.9000 & 0.9000 & 0.7500 & 0.7500 \\
\hline 5 & 0.6500 & 0.5500 & 0.8500 & 0.8500 & 0.7500 & 0.7500 & 0.8000 & 0.8000 & 0.9000 & 0.9000 & 0.8500 & 0.9000 & 0.7500 & 0.8500 & 0.8500 & 0.7000 & 0.7000 \\
\hline 6 & 0.6000 & 0.6000 & 0.9000 & 0.9000 & 0.9000 & 0.8000 & 0.8500 & 0.9500 & 0.8500 & 0.8500 & 0.9000 & 0.8500 & 0.7000 & 0.9000 & 0.9000 & 0.8500 & 0.8500 \\
\hline 7 & 0.6500 & 0.6500 & 0.9500 & 0.9500 & 0.8500 & 0.8500 & 0.9000 & 0.9000 & 1.0000 & 1.0000 & 0.9500 & 1.0000 & 0.7500 & 0.9500 & 0.9500 & 0.8000 & 0.8000 \\
\hline 8 & 0.6500 & 0.6500 & 0.9500 & 0.9500 & 0.8500 & 0.8500 & 0.9000 & 0.9000 & 1.0000 & 1.0000 & 0.9500 & 1.0000 & 0.7500 & 0.9500 & 0.9500 & 0.8000 & 0.8000 \\
\hline 9 & 0.5500 & 0.6500 & 0.9500 & 0.9500 & 0.9500 & 0.8500 & 0.9000 & 1.0000 & 0.9000 & 0.9000 & 0.9500 & 0.9000 & 0.6500 & 0.9500 & 0.9500 & 0.9000 & 0.9000 \\
\hline 10 & 0.6000 & 0.7000 & 1.0000 & 1.0000 & 0.9000 & 0.9000 & 0.9500 & 0.9500 & 0.9500 & 0.9500 & 1.0000 & 0.9500 & 0.7000 & 1.0000 & 1.0000 & 0.8500 & 0.8500 \\
\hline 11 & 0.6000 & 0.6000 & 0.9000 & 0.9000 & 0.8000 & 0.8000 & 0.8500 & 0.8500 & 0.9500 & 0.9500 & 0.9000 & 0.9500 & 0.7000 & 0.9000 & 0.9000 & 0.7500 & 0.7500 \\
\hline 12 & 0.6500 & 0.6500 & 0.9500 & 0.9500 & 0.8500 & 0.8500 & 0.9000 & 0.9000 & 1.0000 & 1.0000 & 0.9500 & 1.0000 & 0.7500 & 0.9500 & 0.9500 & 0.8000 & 0.8000 \\
\hline 13 & 0.6000 & 0.7000 & 1.0000 & 1.0000 & 0.9000 & 0.9000 & 0.9500 & 0.9500 & 0.9500 & 0.9500 & 1.0000 & 0.9500 & 0.7000 & 1.0000 & 1.0000 & 0.8500 & 0.8500 \\
\hline 14 & 0.6000 & 0.6000 & 0.9000 & 0.9000 & 0.8000 & 0.8000 & 0.8500 & 0.8500 & 0.9500 & 0.9500 & 0.9000 & 0.9500 & 0.7000 & 0.9000 & 0.9000 & 0.7500 & 0.7500 \\
\hline 15 & 0.6500 & 0.6500 & 0.9500 & 0.9500 & 0.8500 & 0.8500 & 0.9000 & 0.9000 & 1.0000 & 1.0000 & 0.9500 & 1.0000 & 0.7500 & 0.9500 & 0.9500 & 0.8000 & 0.8000 \\
\hline 16 & 0.6500 & 0.6500 & 0.9500 & 0.9500 & 0.8500 & 0.8500 & 0.9000 & 0.9000 & 1.0000 & 1.0000 & 0.9500 & 1.0000 & 0.7500 & 0.9500 & 0.9500 & 0.8000 & 0.8000 \\
\hline 17 & 0.5500 & 0.6500 & 0.9500 & 0.9500 & 0.9500 & 0.8500 & 0.9000 & 1.0000 & 0.9000 & 0.9000 & 0.9500 & 0.9000 & 0.6500 & 0.9500 & 0.9500 & 0.9000 & 0.9000 \\
\hline 18 & 0.6000 & 0.7000 & 1.0000 & 1.0000 & 0.9000 & 0.9000 & 0.9500 & 0.9500 & 0.9500 & 0.9500 & 1.0000 & 0.9500 & 0.7000 & 1.0000 & 1.0000 & 0.8500 & 0.8500 \\
\hline 19 & $* * * *$ & 0.7000 & 0.6000 & 0.6000 & 0.6000 & 0.7000 & 0.6500 & 0.5500 & 0.6500 & 0.6500 & 0.6000 & 0.6500 & 0.6000 & 0.6000 & 0.6000 & 0.5500 & 0.5500 \\
\hline 20 & 0.3567 & **** & 0.7000 & 0.7000 & 0.7000 & 0.8000 & 0.7500 & 0.6500 & 0.6500 & 0.6500 & 0.7000 & 0.6500 & 0.5000 & 0.7000 & 0.7000 & 0.7500 & 0.7500 \\
\hline 21 & 0.5108 & 0.3567 & **** & 1.0000 & 0.9000 & 0.9000 & 0.9500 & 0.9500 & 0.9500 & 0.9500 & 1.0000 & 0.9500 & 0.7000 & 1.0000 & 1.0000 & 0.8500 & 0.8500 \\
\hline 22 & 0.5108 & 0.3567 & 0.0000 & **** & 0.9000 & 0.9000 & 0.9500 & 0.9500 & 0.9500 & 0.9500 & 1.0000 & 0.9500 & 0.7000 & 1.0000 & 1.0000 & 0.8500 & 0.8500 \\
\hline 23 & 0.5108 & 0.3567 & 0.1054 & 0.1054 & **** & 0.9000 & 0.8500 & 0.9500 & 0.8500 & 0.8500 & 0.9000 & 0.8500 & 0.6000 & 0.9000 & 0.9000 & 0.8500 & 0.8500 \\
\hline 24 & 0.3567 & 0.2231 & 0.1054 & 0.1054 & 0.1054 & **** & 0.9500 & 0.8500 & 0.8500 & 0.8500 & 0.9000 & 0.8500 & 0.7000 & 0.9000 & 0.9000 & 0.8500 & 0.8500 \\
\hline 25 & 0.4308 & 0.2877 & 0.0513 & 0.0513 & 0.1625 & 0.0513 & $* * * *$ & 0.9000 & 0.9000 & 0.9000 & 0.9500 & 0.9000 & 0.7500 & 0.9500 & 0.9500 & 0.9000 & 0.9000 \\
\hline 26 & 0.5978 & 0.4308 & 0.0513 & 0.0513 & 0.0513 & 0.1625 & 0.1054 & **** & 0.9000 & 0.9000 & 0.9500 & 0.9000 & 0.6500 & 0.9500 & 0.9500 & 0.9000 & 0.9000 \\
\hline 27 & 0.4308 & 0.4308 & 0.0513 & 0.0513 & 0.1625 & 0.1625 & 0.1054 & 0.1054 & **** & 1.0000 & 0.9500 & 1.0000 & 0.7500 & 0.9500 & 0.9500 & 0.8000 & 0.8000 \\
\hline 28 & 0.4308 & 0.4308 & 0.0513 & 0.0513 & 0.1625 & 0.1625 & 0.1054 & 0.1054 & 0.0000 & **** & 0.9500 & 1.0000 & 0.7500 & 0.9500 & 0.9500 & 0.8000 & 0.8000 \\
\hline 29 & 0.5108 & 0.3567 & 0.0000 & 0.0000 & 0.1054 & 0.1054 & 0.0513 & 0.0513 & 0.0513 & 0.0513 & $* * * *$ & 0.9500 & 0.7000 & 1.0000 & 1.0000 & 0.8500 & 0.8500 \\
\hline 30 & 0.4308 & 0.4308 & 0.0513 & 0.0513 & 0.1625 & 0.1625 & 0.1054 & 0.1054 & 0.0000 & 0.0000 & 0.0513 & **** & 0.7500 & 0.9500 & 0.9500 & 0.8000 & 0.8000 \\
\hline 31 & 0.5108 & 0.6931 & 0.3567 & 0.3567 & 0.5108 & 0.3567 & 0.2877 & 0.4308 & 0.2877 & 0.2877 & 0.3567 & 0.2877 & $* * * *$ & 0.7000 & 0.7000 & 0.6500 & 0.6500 \\
\hline 32 & 0.5108 & 0.3567 & 0.0000 & 0.0000 & 0.1054 & 0.1054 & 0.0513 & 0.0513 & 0.0513 & 0.0513 & 0.0000 & 0.0513 & 0.3567 & $* * * *$ & 1.0000 & 0.8500 & 0.8500 \\
\hline 33 & 0.5108 & 0.3567 & 0.0000 & 0.0000 & 0.1054 & 0.1054 & 0.0513 & 0.0513 & 0.0513 & 0.0513 & 0.0000 & 0.0513 & 0.3567 & 0.0000 & $* * * *$ & 0.8500 & 0.8500 \\
\hline 34 & 0.5978 & 0.2877 & 0.1625 & 0.1625 & 0.1625 & 0.1625 & 0.1054 & 0.1054 & 0.2231 & 0.2231 & 0.1625 & 0.2231 & 0.4308 & 0.1625 & 0.1625 & **** & 1.0000 \\
\hline 35 & 0.5978 & 0.2877 & 0.1625 & 0.1625 & 0.1625 & 0.1625 & 0.9000 & 0.1054 & 0.2231 & 0.2231 & 0.1625 & 0.2231 & 0.4308 & 0.1625 & 0.1625 & 0.0000 & $* * * *$ \\
\hline
\end{tabular}

Int. J . Agril. Res. Innov. \& Tech. 4 (1): 77-87, J une, 2014 


\section{Genetic differentiation and rate of migration among subdivided population}

Nei's analysis of gene diversity in subdivided populations presented the gene flow $(\mathrm{Nm}+)$ value of 0.000 and the proportion of total genetic diversity

Table 4. Summary of genetic variation statistics across all loci

\begin{tabular}{l|c|c|c|c|c}
\hline Loci & Sample Size & Ht & Hs & Gst & Nm+ \\
\hline OPC 03-1 & 35 & 0.3200 & 0.0000 & 1.0000 & 0.0000 \\
\hline OPC 03-2 & 35 & 0.2024 & 0.0000 & 1.0000 & 0.0000 \\
\hline OPC 03-3 & 35 & 0.1078 & 0.0000 & 1.0000 & 0.0000 \\
\hline OPC 03-4 & 35 & 0.1078 & 0.0000 & 1.0000 & 0.0000 \\
\hline OPC 03-5 & 35 & 0.0555 & 0.0000 & 1.0000 & 0.0000 \\
\hline OPC 03-6 & 35 & 0.1567 & 0.0000 & 1.0000 & 0.0000 \\
\hline OPC 04-1 & 35 & 0.3527 & 0.0000 & 1.0000 & 0.0000 \\
\hline OPC 04-2 & 35 & 0.4800 & 0.0000 & 1.0000 & 0.0000 \\
\hline OPC 04-3 & 35 & 0.0555 & 0.0000 & 1.0000 & 0.0000 \\
\hline OPC 04-4 & 35 & 0.0555 & 0.0000 & 1.0000 & 0.0000 \\
\hline OPC 04-5 & 35 & 0.0000 & 0.0000 & $* * *$ & $* * *$ \\
\hline OPC 04-6 & 35 & 0.3527 & 0.0000 & 1.0000 & 0.0000 \\
\hline OPC 04-7 04-8 & 35 & 0.0555 & 0.0000 & 1.0000 & 0.0000 \\
\hline OPA 01-1 & 35 & 0.0555 & 0.0000 & 1.0000 & 0.0000 \\
\hline OPA 01-2 & 35 & 0.0000 & 0.0000 & $* * * *$ & $* * * *$ \\
\hline OPA 01-3 & 35 & 0.1567 & 0.0000 & 1.0000 & 0.0000 \\
\hline OPA 01-4 & 35 & 0.0000 & 0.0000 & $* * * *$ & $* * * *$ \\
\hline OPA 01-5 & 35 & 0.0000 & 0.0000 & $* * *$ & $* * *$ \\
\hline OPA 01-6 & 35 & 0.0000 & 0.0000 & $* * * *$ & $* * *$ \\
\hline Mean & 35 & 0.0555 & 0.0000 & 1.0000 & 0.0000 \\
\hline St. Dev. & & 0.1285 & 0.0000 & 1.0000 & 0.0000 \\
\hline
\end{tabular}

$\mathrm{H}_{\mathrm{t}}=$ Hardy-Weinberg average heterozygosity expected in sub-population

$\mathrm{H}_{\mathrm{s}}=\mathrm{Hardy}$-Weinberg average heterozygosity obtained in sub-population

$\mathrm{G}_{\mathrm{st}}=\mathrm{Co}$-efficient of gene differentiation

$\mathrm{Nm}=$ Estimate of gene flow from Gst or Gcs. e.g., $\mathrm{Nm}_{\mathrm{m}}=0.5\left(1-\mathrm{Gst}_{\mathrm{st}}\right) / \mathrm{G}_{\mathrm{st}}$

$* * * *=$ Infinity

The number of polymorphic loci is : 15

The percentage of polymorphic loci is : 75.00

\section{UPGMA Dendrogram}

A dendrogram was constructed based on Nei's (1972) genetic distance following the Unweighted Pair Group Method of Arithmetic Means (UPGMA). The 35 genotypes of rice were grouped into 2 main clusters namely cluster 1 and cluster 2 (Fig. 4).

Genotypes Sylhet balam and Mota aman was included in cluster 2. Cluster 2 i.e. Sylhet balam and Mota aman was totally different from other genotypes. So, genetic relationship was not present between cluster 1 genotypes with cluster 2 .

Genotypes belong in cluster 1 were Hati bajore, Moghai balam, Golapi, Malagoti, Sada gotal, Asam binni, Kuchra, Khak shail, Kakua binni, Enghi, J ota balam, Nona bokhra, J amai naru, Khainol, Binadhan-8, Hari, Hamai, Ledra binni, Dakh shail, Rotisail, Moina moti, Genggeng binni, Marish shail, Ghigoj, Jolkumri, Patnai, Piarjat, Mowbinni, Bhute shalot, Lal biroi, Bogi, Kute patnai and Lalanamia.

The genotypes of cluster 1 again divided into two subcluster 1 and sub-cluster 2. Sub-cluster 1 consisted of genotypes Hati bajore, Moghai balam, Golapi, Malagoti, Sada Gotal, Asam binni, Kuchra, Khak shail, Kakua binni, Enghi, J ota balam, Nona bokhra, J amai Naru, Khainol, Binadhan-8, Hari, Hamai, Ledra binni,
(Gst) was 10000. Hardy-Weinberg expectation of average heterozygosity in sub- population (Ht) was 0.1285 , whereas the heterozygosity (Hs) was 0.0000 (Table4) (McDermott and McDonald, 1993). 


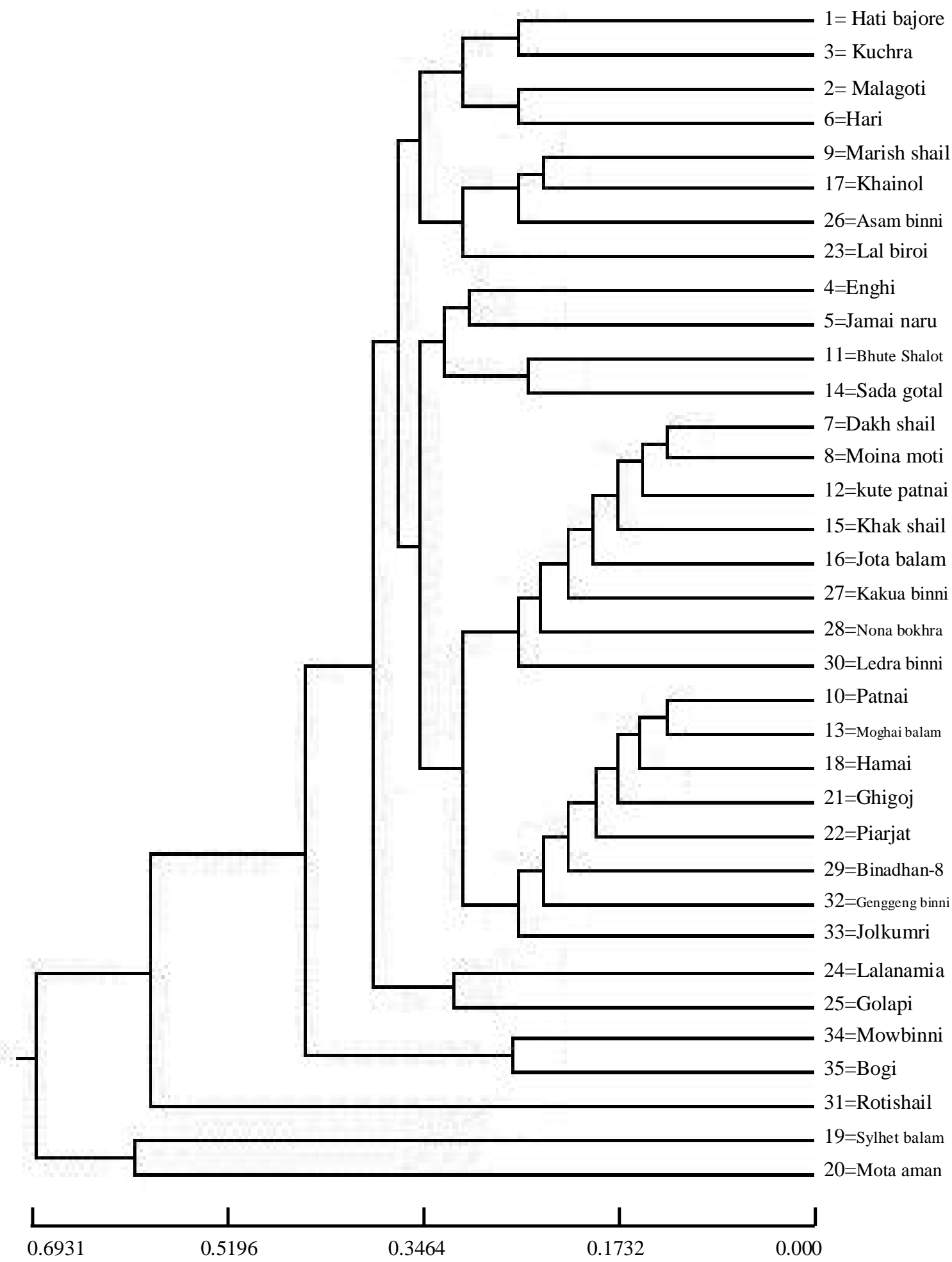

Fig. 4. Unweighted pair group method of arithmetic mean (UPGMA) dendrogram based on Neis's (1972) genetic distance, summarizing data on differentiation in 35 rice genotypes according to RAPD analysis. 


\section{References}

Clarck, A.G. and Lanigan, C.M.S. 1993. Prospects for estimating nucleotide divergence with RAPDS. Mol. Biol. Evol. 10: 1069- 1111.

Mcdermott, J .M. and Mcdonald, B.A. 1993. Gene flow in plant pathosystems. Ann. Rev. Phytopath. 31: 353-373.

Nei, M. 1972. Genetic distance between populations. American Naturalist 106: 283292.

Nei, M. 1973. Analysis of gene diversity in subdivided populations. Proc. Natl. Acad. Sci. 70: 3321-3323.
Skaria, R., Sen, S. and Muneer, P.M.A. 2011. Analysis of genetic variability in rice varieties (Oryza sativa L.) of Kerala using RAPD markers. Genet. Eng. Biotech. J. 24: 1-9.

Weising K., Atkinson, G. and Gardner, C. 1995. Genomic fingerprinting by microsatelliteprimed PCR: a critical evaluation. PCR Methods Applications 4: 249-255.

Yeh, F.C., Yang, R.C., Boyle, T.B.J ., Ye, Z.H. and Mao, J .X. 1997. POPGENE, the user-friendly shareware for population genetic analysis. Molecular Biology and Biotechnology Centre, University of Alberta, Canada. 